Yukawa Institute Kyoto

YITP-00-31

hep-th/0005278

May 2000

\title{
Liouville Integrability of Classical Calogero-Moser Models
}

\author{
S. P. Khastgir and R. Sasaki \\ Yukawa Institute for Theoretical Physics, Kyoto University, \\ Kyoto 606-8502, Japan
}

\begin{abstract}
Liouville integrability of classical Calogero-Moser models is proved for models based on any root systems, including the non-crystallographic ones. It applies to all types of elliptic potentials, i.e. untwisted and twisted together with their degenerations (hyperbolic, trigonometric and rational), except for the rational potential models confined by a harmonic force.
\end{abstract}

In this note we demonstrate the Liouville integrability of classical Calogero-Moser models [1] based on any root systems [2] including the non-crystallographic ones. This applies to models with all possible forms of the potentials, in particular, various types (untwisted and twisted) of elliptic potentials, except for the one with rational potential in the confining harmonic force. The quantum version of this result, restricted to non-elliptic potential cases, is reported in [3].

The proof of Liouville integrability is obtained by combining two known facts: The first is the universal Lax pair of Bordner-Corrigan-Sasaki [4]. This is obtained by unifying the known Lax pairs of various types [5], [6] in terms of representations of Coxeter group, which is 
the symmetry group of Calogero-Moser models. The universal Lax pair provides a complete set of integrals of motion for each Calogero-Moser model with any potential and based on any root system. The second ingredient is a Theorem by Olshanetsky and Perelomov [0, 2] on the structure of the conserved quantities of Calogero-Moser models in general. The latter simply asserts that for the conserved quantities of Calogero-Moser models $\left\{Q_{n}\right\}$ satisfying certain conditions to be listed below, any Poisson brackets among them $\left\{Q_{n}, Q_{m}\right\}$ must vanish.

First let us recapitulate the basic ingredients of the Calogero-Moser models, the Hamiltonian, the Lax pair and conserved quantities in order to set the stage and to introduce notation. A Calogero-Moser model is a Hamiltonian system associated with a root system $\Delta$ of rank $r$. Quantum versions of these models are also integrable, at least for degenerate potential functions [3] for any choice of $\Delta$. The dynamical variables are the coordinates $\left\{q_{j}\right\}$ and their canonically conjugate momenta $\left\{p_{j}\right\}$, with the Poisson brackets

$$
\left\{q_{j}, p_{k}\right\}=\delta_{j k}, \quad\left\{q_{j}, q_{k}\right\}=\left\{p_{j}, p_{k}\right\}=0, \quad j, k=1, \ldots, r .
$$

These will be denoted by vectors in $\mathbf{R}^{r}$

$$
q=\left(q_{1}, \ldots, q_{r}\right), \quad p=\left(p_{1}, \ldots, p_{r}\right)
$$

The Hamiltonian for the Calogero-Moser model is

$$
\mathcal{H}(p, q)=\frac{1}{2} p^{2}+\frac{1}{2} \sum_{\alpha \in \Delta_{+}} g_{|\alpha|}^{2}|\alpha|^{2} V_{|\alpha|}(\alpha \cdot q), \quad \Delta_{+}: \text {set of positive roots, }
$$

in which the real coupling constants $g_{|\alpha|}$ and potential functions $V_{|\alpha|}$ are defined on orbits of the corresponding finite reflection (Coxeter, Weyl) group, i.e. they are identical for roots in the same orbit. The generic potential is elliptic. It is given by Weierstrass' $\wp$ function:

$$
V_{|\alpha|}(\alpha \cdot q)=\wp\left(\alpha \cdot q \mid\left\{2 \omega_{1}, 2 \omega_{3}\right\}\right), \quad \text { for all roots, }
$$

which is called untwisted model. Here the two standard periods of the $\wp$ function are explicitly displayed. In the twisted model [8, 6, 5] the form of the potentials depends on the length of the roots. For long roots, it is the same as above. For short roots

$$
V_{S}(\alpha \cdot q)=\left\{\begin{array}{lc}
\wp\left(\alpha \cdot q \mid\left\{\omega_{1}, 2 \omega_{3}\right\}\right), & \text { for } B_{r}, C_{r}, F_{4} \\
\wp\left(\alpha \cdot q \mid\left\{\frac{2 \omega_{1}}{3}, 2 \omega_{3}\right\}\right), & \text { for } G_{2}
\end{array}\right.
$$


That is, one of the two periods is reduced to be one half for those root systems with $\left|\alpha_{S}\right|^{2} /\left|\alpha_{L}\right|^{2}=1 / 2$ and to one third for $G_{2}$ in which $\left|\alpha_{S}\right|^{2} /\left|\alpha_{L}\right|^{2}=1 / 3$. The other potentials, the hyperbolic, the trigonometric and the rational ones are obtained as one (two) period tends to infinity:

$$
V_{|\alpha|}(\alpha \cdot q)=\frac{a^{2}}{\sinh ^{2} a \alpha \cdot q}, \quad \frac{a^{2}}{\sin ^{2} a \alpha \cdot q}, \quad \frac{1}{(\alpha \cdot q)^{2}}, \quad a \text { : const. }
$$

This then ensures that the Hamiltonian is invariant under reflections of the phase space variables about a hyperplane perpendicular to any root

$$
\mathcal{H}\left(s_{\alpha}(p), s_{\alpha}(q)\right)=\mathcal{H}(p, q), \quad \forall \alpha \in \Delta
$$

with the action of $s_{\alpha}$ on any vector $\gamma \in \mathbf{R}^{r}$ defined by

$$
s_{\alpha}(\gamma)=\gamma-\left(\alpha^{\vee} \cdot \gamma\right) \alpha, \quad \alpha^{\vee} \equiv 2 \alpha /|\alpha|^{2} .
$$

Next we describe the Lax pair and the conserved quantities. Let us choose a set of $\mathbf{R}^{r}$ vectors $\mathcal{R}=\left\{\mu^{(k)} \in \mathbf{R}^{r}, k=1, \ldots, d\right\}$, which form a $d$-dimensional representation of the Coxeter group. That is, they are permuted under the action of the Coxeter group. Then the Lax operators are $d \times d$ dimensional matrices

$$
\begin{aligned}
L(p, q) & =p \cdot \hat{H}+X(q), \quad X(q)=i \sum_{\rho \in \Delta_{+}} g_{|\rho|}(\rho \cdot \hat{H}) x_{|\rho|}\left(\rho \cdot q,\left(\rho^{\vee} \cdot \hat{H}\right) \xi\right) \hat{s}_{\rho}, \\
M(q) & =\frac{i}{2} \sum_{\rho \in \Delta_{+}} g_{|\rho|}|\rho|^{2} y_{|\rho|}\left(\rho \cdot q,\left(\rho^{\vee} \cdot \hat{H}\right) \xi\right) \hat{s}_{\rho}, \quad \text { with } \quad y_{|\rho|}(u, w) \equiv \frac{\partial x_{|\rho|}}{\partial u}(u, w) .
\end{aligned}
$$

consisting of operators $\left\{\hat{H}_{j}\right\}$ and $\left\{\hat{s}_{\rho}\right\}$. Their matrix elements are defined by:

$$
\left(\hat{H}_{j}\right)_{\mu \nu}=\mu_{j} \delta_{\mu \nu}, \quad\left(\hat{s}_{\rho}\right)_{\mu \nu}=\delta_{\mu, s_{\rho}(\nu)}=\delta_{\nu, s_{\rho}(\mu)}, \quad \mu, \nu \in \mathcal{R} .
$$

The $L$ and $M$ operators are Coxeter covariant:

$$
L\left(s_{\rho}(p), s_{\rho}(q)\right)_{\mu \nu}=L(p, q)_{\mu^{\prime} \nu^{\prime}}, M\left(s_{\rho}(q)\right)_{\mu \nu}=M(q)_{\mu^{\prime} \nu^{\prime}}, \quad \mu^{\prime} \equiv s_{\rho}(\mu), \nu^{\prime} \equiv s_{\rho}(\nu),
$$

and they contain a spectral parameter $\xi$ [9] which enters through functions $x_{|\alpha|}(u, w)$. For the untwisted model it is $(\wp(u) \equiv-d \zeta(u) / d u$ and $\zeta(u) \equiv d \log \sigma(u) / d u)$

$$
x_{|\alpha|}(u, w)=\frac{\sigma(w-u)}{\sigma(w) \sigma(u)}, \quad \text { for all roots, }
$$


in which the dependence on the standard periods is suppressed. For long roots in the twisted model, it is the same as above and for short roots

$$
x_{S}(u, w)=\left\{\begin{array}{lc}
\frac{\sigma\left(w / 2-u \mid\left\{\omega_{1}, 2 \omega_{3}\right\}\right)}{\sigma\left(w / 2 \mid\left\{\omega_{1}, 2 \omega_{3}\right\}\right) \sigma\left(u \mid\left\{\omega_{1}, 2 \omega_{3}\right\}\right)} & \text { for } B_{r}, C_{r}, F_{4} \\
\frac{\sigma\left(w / 3-u \mid\left\{2 \omega_{1} / 3,2 \omega_{3}\right\}\right)}{\sigma\left(w / 3 \mid\left\{2 \omega_{1} / 3,2 \omega_{3}\right\}\right) \sigma\left(u \mid\left\{2 \omega_{1} / 3,2 \omega_{3}\right\}\right)} & \text { for } G_{2} .
\end{array}\right.
$$

Near the origin $q=0$, or $u=0$, the $u$ dependence of the various $x$ functions is universal:

$$
x_{|\alpha|}(u, w)=\frac{1}{u}\left(1-u \zeta_{c}(w / c)\right), \quad c=1,2,3, \quad|u| \ll 1, \quad \zeta_{c}(u) \equiv \zeta\left(u \mid\left\{2 \omega_{1} / c, 2 \omega_{3}\right\}\right),
$$

which is the same as that of the rational potential except for the spectral parameter dependence:

$$
x_{|\alpha|}(u, w)=\frac{1}{u}\left(1-\frac{u}{w}\right), \quad \text { for rational potential. }
$$

The canonical equations of motion can be cast into the Lax form

$$
\dot{L}=[L, M]
$$

which implies that the traces of $L$ are conserved:

$$
Q_{n}(p, q) \equiv \operatorname{Tr}\left(L^{n}(p, q)\right)=\sum_{\mu \in \mathcal{R}}\left(L^{n}\right)_{\mu \mu}, \quad \frac{d}{d t} Q_{n}=\left\{Q_{n}, \mathcal{H}\right\}=0, \quad n=1,2, \ldots,
$$

The independent ones appear at such power $n$ that it is $1+$ exponent of the root system. This is summarised in the following Table I:

\begin{tabular}{||c|l||c|l||}
\hline$\Delta$ & $f_{j}=1+$ exponent & $\Delta$ & $f_{j}=1+$ exponent \\
\hline$A_{r}$ & $2,3,4, \ldots, r+1$ & $E_{8}$ & $2,8,12,14,18,20,24,30$ \\
\hline$B_{r}$ & $2,4,6, \ldots, 2 r$ & $F_{4}$ & $2,6,8,12$ \\
\hline$C_{r}$ & $2,4,6, \ldots, 2 r$ & $G_{2}$ & 2,6 \\
\hline$D_{r}$ & $2,4, \ldots, 2 r-2 ; r$ & $I_{2}(m)$ & $2, m$ \\
\hline$E_{6}$ & $2,5,6,8,9,12$ & $H_{3}$ & $2,6,10$ \\
\hline$E_{7}$ & $2,6,8,10,12,14,18$ & $H_{4}$ & $2,12,20,30$ \\
\hline
\end{tabular}

Table I: The degrees $f_{j}$ in which independent Coxeter invariant polynomials exist.

Thanks to the availability of various representations for the Lax pair, a complete set of independent conserved quantities $\left\{Q_{n}(p, q)\right\}$ are obtained as traces of certain powers of $L$. The independence of the conserved quantities can be easily verified by considering the free limit, i.e. $g_{|\rho|}=0$. They have the following properties: 
1. Coxeter invariance as a consequence of (11):

$$
Q_{n}\left(s_{\rho}(p), s_{\rho}(q)\right)=Q_{n}(p, q), \quad \forall \rho \in \Delta .
$$

2. $Q_{n}(p, q)$ is a homogeneous polynomial of degree $n$ in variables $\left(p_{1}, \ldots, p_{r}, x_{|\alpha|}(\rho \cdot q, w)\right)$, in which $w$ is proportional to the spectral parameter.

3. Scaling property for those of rational potential models:

$$
{ }^{R} Q_{n}\left(\kappa^{-1} p, \kappa q\right)=\kappa^{-n R} Q_{n}(p, q)+\text { sub-leading terms, }
$$

as a consequence of the above point and (15).

4. For the other types of potential, the asymptotic behaviour near the origin:

$$
Q_{n}(p, q)={ }^{R} Q_{n}(p, q)(1+\mathcal{O}(|q|)), \quad \text { for } \quad|q| \rightarrow 0 .
$$

We need to show the vanishing of

$$
J_{l m} \equiv\left[Q_{l}, Q_{m}\right],
$$

which is a polynomial in $\{p\}$ of degree $s$

$$
s<l+m,
$$

since the leading powers in $\{p\}, p^{l}\left(p^{m}\right)$ in $Q_{l}\left(Q_{m}\right)$, having constant coefficients, commute with each other. Let us decompose $J_{l m}$ into the leading part and the rest:

$$
J_{l m}=J_{l m}^{0}+J_{l m}^{r e s t}, \quad J_{l m}^{0}=\sum c^{j_{1}, \ldots, j_{s}}(q) p_{j_{1}} \ldots p_{j_{s}}
$$

and $J_{l m}^{r e s t}$ is a polynomial in $\{p\}$ of degree less than $s$. The Theorem by Olshanetsky and Perelomov [7] (Lemma 2, section 4) states:

If the leading coefficients $c^{j_{1}, \ldots, j_{s}}(q)$ are not constant, then $J_{l m}=0$.

Thus the involution is proved. The argument goes as follows. From Jacobi identity and conservation $\left\{\mathcal{H}, Q_{l(m)}\right\}=0$, we obtain

$$
\left\{\mathcal{H}, J_{l m}\right\}=0 .
$$


Considering the explicit form of the Hamiltonian (3), the leading (i.e. of degree $s+1$ in $\{p\}$ ) part of $\left\{\mathcal{H}, J_{l m}\right\}$ comes only from the free part

$$
\left\{p^{2}, J_{l m}^{0}\right\}
$$

and it vanishes if the following conditions are satisfied:

$$
\sum_{\sigma} \frac{\partial}{\partial q_{t}} c^{k_{1}, \ldots, k_{s}}(q)=0
$$

where the sum is taken over all permutations of indices $\sigma\left(t, k_{1}, \ldots, k_{s}\right)=\left(j_{1}, \ldots, j_{s+1}\right)$. In 110 it is proved (Lemma 2.5, p. 407) that the system (25) has only polynomial solutions. For rational potential models the scaling property of the leading terms tells that $c^{k_{1}, \ldots, k_{s}}(\kappa q)=$ $\kappa^{s-l-m} c^{k_{1}, \ldots, k_{s}}(q)$. Since $s<l+m$ (22), it follows that the only polynomial solution satisfying the condition is the null polynomial. Thus we obtain $c^{j_{1}, \ldots, j_{s}}(q)=0 \Rightarrow J_{l m}^{0}=0$ and $J_{l m}=0$. The same results follow for the other types of potentials by considering the asymptotic behaviour for $|q| \rightarrow 0$. Thus the involution of all the conserved quantities $\left\{Q_{n}\right\}$ is proved for the models with rational, hyperbolic, trigonometric and various types of elliptic potentials for all root systems.

It would be interesting to relate this type of argument to other approaches to the involution properties, for example, those of the classical $r$-matrices for elliptic models by Sklyanin and others [11- 12.

We thank A. J. Pocklington for useful discussion. This work is partially supported by the Grant-in-aid from the Ministry of Education, Science and Culture, priority area (\#707) "Supersymmetry and unified theory of elementary particles". S. P. K. is supported by the Japan Society for the Promotion of Science.

\section{References}

[1] F. Calogero, "Solution of the one-dimensional $N$-body problem with quadratic and/or inversely quadratic pair potentials", J. Math. Phys. 12 (1971) 419-436; B. Sutherland, "Exact results for a quantum many-body problem in one-dimension. II", Phys. Rev. A5 (1972) 1372-1376; J. Moser, "Three integrable Hamiltonian systems connected with isospectral deformations", Adv. Math. 16 (1975) 197-220; J. Moser, "Integrable systems of non-linear evolution equations", in Dynamical Systems, Theory and Applications; J. 
Moser, ed., Lecture Notes in Physics 38 (1975), Springer-Verlag; F. Calogero, C. Marchioro and O. Ragnisco, "Exact solution of the classical and quantal one-dimensional many body problems with the two body potential $V_{a}(x)=g^{2} a^{2} / \sinh ^{2} a x$ ", Lett. Nuovo Cim. 13 (1975) 383-387; F. Calogero, "Exactly solvable one-dimensional many body problems", Lett. Nuovo Cim. 13 (1975) 411-416.

[2] M. A. Olshanetsky and A. M. Perelomov, "Completely integrable Hamiltonian systems connected with semisimple Lie algebras", Inventions Math. 37 (1976), 93-108; "Quantum integrable systems related to Lie algebras", Phys. Rep. 94 (1983) 313-404.

[3] S. P. Khastgir, A. J. Pocklington and R. Sasaki, "Quantum Calogero-Moser Models: Integrability for all Root Systems", Kyoto-preprint YITP-00-24, hep-th/0005277.

[4] A. J. Bordner, E. Corrigan and R. Sasaki, "Generalised Calogero-Moser models and universal Lax pair operators", Prog. Theor. Phys. 102 (1999) 499-529, hep-th/9905011.

[5] A. J. Bordner, E. Corrigan and R. Sasaki, "Calogero-Moser models I: a new formulation", Prog. Theor. Phys. 100 (1998) 1107-1129, hep-th/9805106; A. J. Bordner, R. Sasaki and K. Takasaki, "Calogero-Moser models II: symmetries and foldings", Prog. Theor. Phys. 101 (1999) 487-518, hep-th/9809068; A. J. Bordner and R. Sasaki, "Calogero-Moser models III: elliptic potentials and twisting", Prog. Theor. Phys. 101 (1999) 799-829, hep-th/9812232; S. P. Khastgir, R. Sasaki and K. Takasaki, "Calogero-Moser Models IV: Limits to Toda theory", Prog. Theor. Phys. 102 (1999), 749-776, hep-th/9907102.

[6] E. D'Hoker and D.H. Phong, "Calogero-Moser Lax pairs with spectral parameter for general Lie algebras", Nucl. Phys. B530 (1998) 537-610, hep-th/9804124.

[7] M. A. Olshanetsky and A. M. Perelomov, "Quantum systems related to root systems, and radial parts of Laplace operators", Funct. Anal. Appl. 12 (1977)121-128.

[8] V.I. Inozemtsev, "Lax representation with spectral parameter on a torus for integrable particle systems", Lett. Math. Phys. 17 (1989) 11-17.

[9] I. M. Krichever, "Elliptic solutions of the Kadomtsev-Petviashvili equation and integrable systems of particles", Funct. Anal. Appl. 14 (1980) 282-289. 
[10] F. A. Berezin, "Laplace operators on semisimple Lie groups", Tr. Mosk. Mat. Ob-va, 6 (1957) 371-463.

[11] J. Avan and M. Talon, "Classical R-matrix structure for the Calogero model", Phys. Lett. B303 (1933) 33-37, hep-th/9210128; J. Avan, O. Babelon and M. Talon, "Construction of the classical R matrices for the Toda and Calogero models", Alg. Anal. 6 (1994) 67-89, hep-th/9306102.

[12] E. K. Sklyanin, "Dynamical $r$-matrices for the elliptic Calogero-Moser model", Alg. Anal. 6 (1994) 227, hep-th/9308060; H. W. Braden and T. Suzuki, " $R$-matrices for the elliptic Calogero-Moser model", Lett. Math. Phys. 30 (1994) 147-158, hep-th/9309033. 